\title{
Investigations into Capacity of MIMO Ad Hoc Network Including Effects of Antenna Mutual Coupling
}

\author{
Xia LIU ${ }^{1}$, Marek E. BIALKOWSKI ${ }^{2}$, Feng WANG ${ }^{2}$, Konglit HUNCHANGSITH ${ }^{2}$ \\ ${ }^{1}$ Correspondant author, School of ITEE, the University of Queensland, Brisbane, Australia \\ ${ }^{2}$ School of ITEE, the University of Queensland, Brisbane, Australia \\ Email: \{xialiu, meb, fwang, konglit\}@itee.uq.edu.au \\ Received August 6, 2009; revised September 27, 2009; accepted September 30, 2009
}

\begin{abstract}
This paper reports on investigations into capacity of ad hoc network whose nodes are equipped with multiple element antennas (MEAs). The investigation of this multi-user Multiple Input Multiple Output (MIMO) system takes into account mutual coupling (MC) in addition to spatial correlation that is present in array antennas. A closed-form expression for an upper bound of mutual information (capacity) of MIMO ad hoc network is derived. An optimal signal transmission scheme is proposed to maximize the MIMO ad hoc network capacity. Simulation results for capacity of non-optimized and optimized cases of signal transmission are presented.
\end{abstract}

Keywords: MIMO, Ad Hoc Network, Channel Capacity, Mutual Information, Mutual Coupling, Spatial Correlation

\section{Introduction}

In recent years, the signal transmission technique employing multiple element antennas (MEAs) at two sides of a communication link has shown a great potential to significantly improve the transmission quality of wireless communication systems without the need for extra operational frequency bandwidth [1-3]. Because of this attribute, the multiple-input multiple-output (MIMO) technique is envisaged for the next generation of mobile communications.

Benefits of MIMO technique over the traditional single-input single-output (SISO) signal transmission with respect to the capacity and Bit Error Rate (BER) have been demonstrated for single-user (peer-to-peer) scenarios. An ultimate goal is to prove its advantages for multi-user wireless communication networks.

Wireless networks are usually formed by cells each with a base station (BS) and many mobile stations (MS). Mobile terminals communicate with the base station (BS) which organizes multiple-access to the intended MS users. To implement the MIMO concept for such a standard mobile communication system, MS and BS have to be equipped with multiple element antennas and suitable signal processing algorithms.

Nowadays, there is an increased interest in mobile ad hoc networks in which pairs of mobile nodes communicate directly with each other without the involvement of BS. The aim is to improve spectral efficiency and capacity of such networks [4,5]. In order to form a MIMO ad hoc network, multiple element antennas with associated signal processing algorithms have to be implemented at mobile nodes.

It is known that MIMO systems operate well in rich scattering environments. This is because such environments support virtual multiple channels which are statistically independent. Finite spacing of multiple element antennas introduces spatial correlation which decreases the effective degree of freedom (EDOF) [6] and thus reduces the MIMO system capacity. The adverse effect of spatial correlation has been pointed out both for single and multiple user scenarios [6-8]. The finite antenna spacing in array antennas is also responsible for mutual coupling which adversely affects power transmission and reception. The mutual coupling effect is especially pronounced in tightly spaced arrays. Because there is a considerable demand for compact size MS terminals, the effect of mutual coupling cannot be neglected and has to be taken into account while assessing the MIMO link performance. The effect of mutual coupling in MIMO systems for the case of peer to peer communication was addressed via simulations and measurements in [9-12] where it was demonstrated that in some cases the mutual coupling could reduce spatial correlation and improve the channel capacity.

In this paper, we report on investigations into the capacity of MIMO ad hoc network. The investigations take into account both the spatial correlation and the mutual coupling that are present in array antennas. A closedform expression for an upper bound of the mutual infor- 
mation (capacity) of MIMO ad hoc network taking into account spatial correlation and mutual coupling is derived. Assuming that the channel state information (CSI) is known both by the receiving node and the transmitting node, a cooperative communication is proposed to fight multi-user interference. It is shown that using the cooperative communication a higher MIMO channel capacity is achieved in comparison with the case when the CSI acquired at the receiving node is not shared with the other nodes of the network.

The rest of the paper is organized as follows. Section 2 describes the network configuration and the channel model that takes into account mutual coupling in transmitting and receiving array antennas. The capacity of non-cooperative and co-operative ad hoc network is given in Section 3. Section 4 presents the simulation results and Section 5 concludes the paper.

\section{System Configuration and Channel Model}

\subsection{System Configuration}

In this paper, a narrowband MIMO ad hoc network consisting of $N$ nodes is considered. Its configuration is shown in Figure 1. Each node is assumed to use a transceiver equipped with a uniform linear array of $M_{r t}$ vertically polarized wire dipole antennas. The choice of wire dipoles is justified by the fact that there are analytical solutions for the spatial correlation and mutual coupling for such type of array antenna. The network operates in a scattering environment in which each node is surrounded by scattering objects uniformly distributed within a circle, as shown in Figure 1.

A channel formed between a pair of node $i$ and the transmitting node $j$ is characterized by the $M_{r t} \times M_{r t}$ complex channel matrix $\mathbf{H}_{i j}$ whose elements are given by complex transmission coefficients between the individual antenna elements at nodes $j$ and $i$.

Under these assumptions, the signal received at node $i$ is described by the following equation,

$$
\mathbf{y}_{i}=\mathbf{H}_{i j} \mathbf{s}_{j}+\sum_{k=1, k \neq j}^{N-1} \mathbf{H}_{i k} \mathbf{s}_{k}+\mathbf{n}_{i}
$$

The first term in (1) represents the signal received from the desired node as given by the channel matrix $\mathbf{H}_{i j}$; and the $M_{r t} \times 1$ transmitted signal vector $\mathbf{s}_{j}$. The second term is the sum of co-channel interference and $\mathbf{n}_{i}$ is the $M_{r t}$ X 1 noise vector. The co-channel interferenceplus-noise (IPN) can be expressed as,

$$
\mathbf{N}_{i}=\sum_{k=1, k \neq j}^{N-1} \mathbf{H}_{i k} \mathbf{s}_{k}+\mathbf{n}_{i}
$$

The correlation of IPN is given by the following expression,

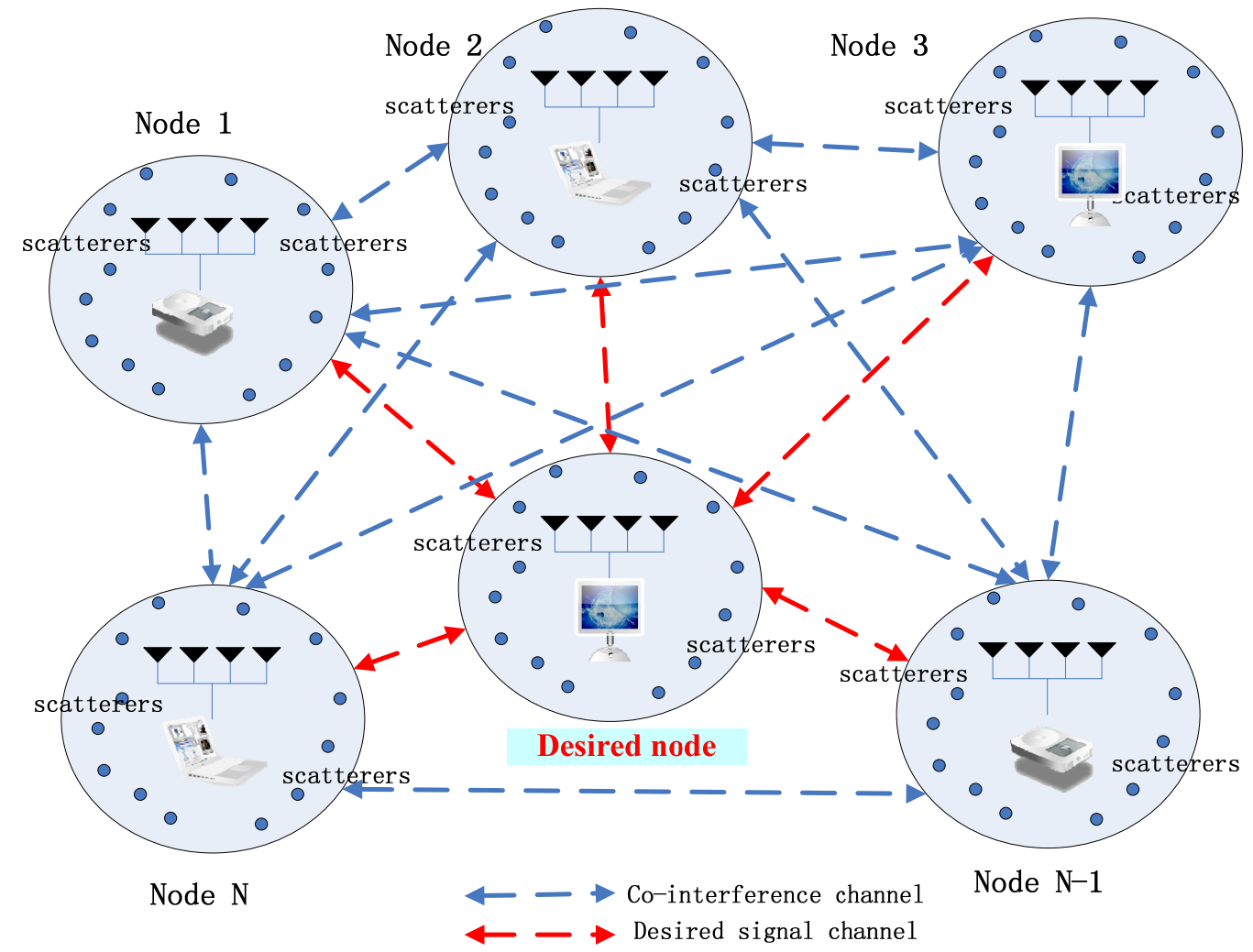

Figure 1. MIMO ad hoc network. 


$$
R_{N_{i}}=E\left\{N_{i} N_{i}^{H}\right\}=\sum_{k=1, k \neq j}^{N-1} \mathbf{H}_{i k} Q_{k} \mathbf{H}_{i k}^{H}+\sigma_{n}^{2} I_{M_{r t}}
$$

where $Q_{k}=E\left\{s_{k} s_{k}{ }^{H}\right\}$ is the data covariance matrix representing the signal transmission scheme from the $k$-th inference node to node $i . E\{\}$ denotes the statistical expectation.

For the case of non-optimized ad hoc network, only receivers have the knowledge of CSI. In such a case, the best strategy for the nodes is to transmit equal power over the individual transmitting antenna elements. In this case, the $Q_{i k}$ of the $k$-th interference node can be expressed as:

$$
\begin{aligned}
& Q_{i k}=\frac{p_{k}}{N_{t}} I, \\
& \text { subject to }: P_{I}=\sum_{k=1}^{N-1} p_{k}, p_{1}=p_{2}=\ldots=p_{k}
\end{aligned}
$$

where $p_{k}$ is the transmit power from node $k$ and $P_{I}$ is the total transmitted power of all interferences.

By using (4), Equation (3) can be rewritten as:

$$
R_{N_{i}}=\frac{P_{I}}{N-1} \sum_{k=1, k \neq j}^{N-1} \mathbf{H}_{i k} \mathbf{H}_{i k}^{H}+\sigma_{n}^{2} I_{M_{r t}}
$$

The correlation of received signal at node $i$ that is transmitted from the desired node is obtained as:

$$
R_{i}^{y}=E\left\{y_{i} y_{i}{ }^{H}\right\}=\mathbf{H}_{i j} Q_{j} \mathbf{H}_{i j}{ }^{H}+R_{N_{i}}
$$

in which $Q_{j}=E\left\{s_{j} s_{j}{ }^{H}\right\}$ is the data covariance matrix representing the signal transmission scheme from node $j$ to node $I$, which is also the mutual information for the channel formed between the desired node $j$ and node $i$. $\operatorname{tr}\left\{Q_{j}\right\}=P_{j}$ and $P_{j}$ is the total transmitted signal power from the desired node.

The capacity of the system can be improved by optimizing the signal transmission scheme. The optimization process requires the knowledge of CSI by transmitters which is usually obtained by a feedback loop from receivers. In this case, the new data correlation matrix, subject to constraint of total transmitted power $\operatorname{tr}\left\{Q_{j}\right\}=P_{j}$, has to be worked out.

\subsection{Channel Model Neglecting Mutual Coupling}

In the undertaken investigations, a narrow-band flat block-fading MIMO channel is assumed between the network nodes. For each pair of nodes, the channel matrix $(\mathbf{H})$ can be used to describe the channel properties. Its entries depend on the signal propagation environment and properties of the antenna arrays used at the two sides of communication link.

In the initial stage, the Kronecker representation of channel $[13,14]$ neglecting the effects of mutual coupling is assumed. In this representation, the transmitter and receiver correlations are separable and the channel matrix $\mathbf{H}$ (for brevity the subscripts are dropped here) is represented as:

$$
\mathbf{H}=R_{R}^{1 / 2} G_{H} R_{T}^{1 / 2}
$$

where $G_{H}$ is the matrix including identical independent distributed (i.i.d) Gaussian entries with zero mean and unit variance, and $R_{R}$ and $R_{T}$ are the spatial correlation matrices at the receiver and transmitter, respectively. The channel correlation is expressed as,

$$
R_{H}=E\left\{\mathbf{H} \mathbf{H}^{H}\right\}
$$

Because each node includes vertically polarized wire dipole antennas and the scattering environment is represented by circles of uniformly distributed scattering objects surrounding nodes as shown in Fig.1, the spatial correlation matrix elements can be obtained using the Clark's model as given by.

$$
\rho_{l, m}^{R(T)}=J_{0}\left(\kappa d_{l, m}\right)
$$

where $J_{0}$ stands for the zero-order Bessel function, $\kappa$ is a wave number and $d_{l m}$ is the distance between elements $l$ and $m$ of the uniform array antenna.

The correlation matrices $R_{T}$ and $R_{R}$ can be generated using (9) as,

$$
R_{R(T)}=\left[\begin{array}{ccc}
\rho_{1,1}^{R(T)} & \cdots & \rho_{1, M_{r t}}^{R(T)} \\
\vdots & \ddots & \vdots \\
\rho_{M_{r t}, j}^{R(T)} & \cdots & \rho_{M_{r t}, M_{r t}}^{R(T)}
\end{array}\right]
$$

Having defined $R_{T}$ and $R_{R}$, the channel matrix for each pair of nodes $(\mathbf{H})$ can be calculated using Equation (7).

\subsection{Channel Model Including Mutual Coupling}

The mutual coupling in an array of collinear side-by-side wire dipoles can be modeled using the theory described in [16]. Assuming the array is formed by $M_{r t}$ wire dipoles, the mutual matrix can be calculated using the following expression

$$
\boldsymbol{C}=\left(\mathrm{Z}_{A}+\mathrm{Z}_{T}\right)\left(\mathbf{Z}+\mathrm{Z}_{T} \boldsymbol{I}_{M_{r t}}\right)^{-1}
$$

where $\mathbf{Z}$ is the impedance matrix, $Z_{\mathrm{A}}$ is the element input impedance in isolation, e.g. when the wire dipole is $\lambda / 2$, its value is $Z_{A}=73+j 42.5[\Omega] ; Z_{T}$ is impedance of the receiver chosen as the complex conjugate of $Z_{\mathrm{A}}$ to obtain the impedance match for best power transfer.

The impedance matrix $\mathbf{Z}$ is given by

$$
\mathbf{Z}=\left[\begin{array}{cccc}
Z_{A}+Z_{T} & Z_{12} & \cdots & Z_{1 M_{r t}} \\
Z_{21} & Z_{A}+Z_{T} & \cdots & Z_{2 M_{r t}} \\
\vdots & \vdots & \ddots & \vdots \\
Z_{M_{r t} 1} & Z_{M_{r t} 2} & \cdots & Z_{A}+Z_{T}
\end{array}\right]
$$


Note that this expression provides the circuit representation for mutual coupling in array antennas. It is valid for single mode antennas. Wire dipoles fall into this category.

For the side-by-side configuration of dipoles having length $l$ equal to $0.5 \lambda$, the expressions for $\left\{Z_{m n}\right\}$ can be adapted from $[16,17]$ and are rewritten here as,

$$
\mathbf{Z}_{m n}=\left\{\begin{array}{c}
30\left[0.5772+\ln (2 \kappa l)-C_{i}(2 \kappa l)\right] \\
+j\left[30 S_{i}(2 \kappa l)\right], \quad m=n \\
30\left[2 C_{i}\left(u_{0}\right)-C_{i}\left(u_{1}\right)-C_{i}\left(u_{2}\right)\right] \\
\quad-j\left[30\left(2 S_{i}\left(u_{0}\right)-S_{i}\left(u_{1}\right)-S_{i}\left(u_{2}\right)\right)\right], m \neq n
\end{array}\right.
$$

where $\kappa$ is the wave number equal to $2 \pi / \lambda$,

$$
\begin{aligned}
& u_{0}=\kappa d_{h}, \\
& u_{1}=\kappa\left(\sqrt{d_{h}^{2}+l^{2}}+l\right) \\
& u_{2}=\kappa\left(\sqrt{d_{h}{ }^{2}+l^{2}}-l\right)
\end{aligned}
$$

$d_{h}$ is the horizontal distance between the two dipole antenna elements. $\mathrm{Ci}(\mathrm{u})$ and $\mathrm{Si}(\mathrm{u})$ are the cosine and sine integrals, respectively. They are given as,

$$
\begin{aligned}
& C_{i}(u)=\int_{\infty}^{u}(\cos (x) / x) d x \\
& S_{i}(u)=\int_{0}^{\infty}(\sin (x) / x) d x
\end{aligned}
$$

The expression for the coupling matrix (11) can be used both at the transmitting and receiving nodes to modify the channel matrices and the correlation matrices. When the mutual coupling is included, the channels matrices $\mathbf{H}$ shown in (7) has to be modified to the new form given by

$$
\mathbf{H}^{\prime}=C_{R} \mathbf{H} C_{T}
$$

where the mutual coupling matrices are calculated using (11).

Similarly, the receiving and transmitting correlation matrices are modified using

$$
\begin{aligned}
& R_{R m u}=C_{R} R_{R}^{\frac{1}{2}} \\
& R_{T m u}=R_{T}^{\frac{1}{2}} C_{T}
\end{aligned}
$$

\section{Capacity of MIMO Ad Hoc Network}

\subsection{Channel Capacity between Individual Nodes}

Having derived the channel matrices between the individual notes for the cases without and with mutual coupling, the next step is to obtain the channel capacities. The mutual information of the channel formed between node $i$ and the desired transmitting node $j$ of MIMO ad-hoc network can be obtained in a similar way as for the case of a multi-access MIMO system [15] and is given as:

$$
\begin{aligned}
& I\left(y_{i} ; s_{j} \mid s_{k, k \neq j}\right) \\
& \leq I\left(y_{i} ; s_{j} \mid s_{k, k \neq j}, \mathbf{H}_{i j}, \mathbf{H}_{i k}\right) \\
& =h\left(y_{i} \mid s_{k, k \neq j}, \mathbf{H}_{i j}, \mathbf{H}_{i k}\right)-h\left(y_{i} \mid s_{k, k \neq j}, s_{j}, \mathbf{H}_{i j}, \mathbf{H}_{i k}\right) \\
& =h\left(y_{i} \mid s_{k, k \neq j}, \mathbf{H}_{i j}, \mathbf{H}_{i k}\right)-h\left(N_{i}\right)
\end{aligned}
$$

The upper bound for capacity can be obtained from the following,

$$
\begin{aligned}
& I\left(y_{i} ; s_{j} \mid s_{k, k \neq j}\right) \\
& \leq E\left\{\log _{2} \operatorname{det}\left[\pi e\left(\mathbf{H}_{i j} Q_{j} \mathbf{H}_{i j}{ }^{H}+R_{N_{i}}\right)\right]-\log _{2} \operatorname{det}\left(\pi e R_{N_{i}}\right)\right\} \\
& \left.=E\left\{\log _{2} \operatorname{det}\left(I_{M_{r t}}+\frac{R_{N_{I}}^{-1} \mathbf{H}_{i j} Q_{j} \mathbf{H}_{i j}{ }^{H}}{\sigma_{n}^{2}}\right)\right]\right\} \\
& \left.=E\left\{\log _{2} \operatorname{det}\left(I_{M_{r t}}+\frac{Q_{j}}{\sigma_{n}^{2}}\left({R_{N_{i}}^{-\frac{1}{2}}}_{\mathbf{H}_{i j}}\right)\left(R_{N_{i}}^{-\frac{1}{2}} \mathbf{H}_{i j}\right)^{H}\right)\right]\right\}
\end{aligned}
$$

where $\sigma_{n}^{2}$ is the noise power.

When both the Kronecker representation (7) and the mutual coupling (11) are included in the MIMO channel model, the expression (20) for the upper bound of mutual information of the channel formed between node $i$ and the desired transmitting node $j$ of MIMO ad-hoc network is modified to:

$$
\begin{aligned}
& I\left(y_{i} ; s_{j} \mid s_{i k}\right)= \\
& E\left\{\operatorname { l o g } _ { 2 } \operatorname { d e t } \left[I_{M_{r t}}\right.\right. \\
& \left.\left.\quad+\frac{Q_{j}}{\sigma_{n}^{2}}\left(R_{N_{i}}^{-\frac{1}{2}} R_{R m u}^{j} G_{H_{i j}} R_{T m u}^{j}\right)\left(R_{N_{i}}^{-\frac{1}{2}} R_{R m u}^{j} G_{H_{i j}} R_{T m u}^{j}\right)^{H}\right]\right\}
\end{aligned}
$$

The correlation of IPN with mutual coupling is given by (22),

$$
R_{N_{i}}^{m u}=\frac{P_{I}}{N} \sum_{i=1}^{N-1}\left(R_{R m u}^{i k} G_{H_{i k}} R_{T m u}^{i k}\right)\left(R_{R m u}^{i k} G_{H_{i k}} R_{T m u}^{i k}\right)^{H}+\sigma_{n}^{2} I_{M_{r t}}
$$

The expression for the upper bound of mutual information (21) is given for a specified signal transmission scheme as described by the data covariance matrix $Q_{j}=$ $E\left\{s_{j} s_{j}^{H}\right\}$.

The mutual information can be maximized by optimizing the signal transmission scheme subject to $\operatorname{tr}\left\{Q_{j}\right\}=P_{j}$. As a a result, the capacity between the $i$-th node and the desired transmitting node for the optimized signal transmission scheme is given by

$$
\begin{aligned}
C_{i}= & \max _{t r\left(Q_{j} / \sigma_{n}^{2}\right) \leq P_{j} / \sigma_{n}^{2}} E\left\{\operatorname { l o g } _ { 2 } \operatorname { d e t } \left[I_{M_{n}}\right.\right. \\
& \left.\left.+\frac{Q_{j}}{\sigma_{n}^{2}}\left(R_{N_{i}}^{-\frac{1}{2}} R_{R m u}^{i j} G_{H_{i j}} R_{T m u}^{i j}\right)\left(R_{N_{i}}^{-\frac{1}{2}} R_{R m u}^{i j} G_{H_{i j}} R_{T m u}^{i j}\right)^{H}\right]\right\}
\end{aligned}
$$

\subsection{Capacity of Non-Cooperative Network}

The capacity of ad hoc network that uses the optimized transmission scheme between individual nodes can be 
written as the sum of individual optimized link capacities $C_{i}$ and therefore can be expressed as,

$$
\begin{aligned}
& C=\sum_{i=1}^{N} C_{i} \\
& =\sum_{i=1}^{N} E\left\{\log _{2} \operatorname{det}\left[I_{M_{n t}}+\frac{Q_{j}}{\sigma_{n}^{2}}\left(R_{N_{i}}^{-\frac{1}{2}} R_{R m u}^{i j} G_{H_{i j}} R_{T m u}^{i j}\right)\left(R_{N_{i}}^{-\frac{1}{2}} R_{R m u}^{i j} G_{H_{i j}} R_{T m u}^{i j}\right)^{H}\right]\right\}
\end{aligned}
$$

\subsection{Capacity of Cooperative Network}

The capacity of ad hoc network can be further improved by assuming cooperation between individual nodes. To accomplish this, first the desired transmitting node has to have the knowledge of channel state information (CSI) from all the remaining nodes of the network. This means that all of the complex channel matrices $\mathbf{H}_{i j}$ are perfectly known to the transmitting nodes. In practice, the required CSI is first acquired by receivers by using the training sequences between the pairs of nodes and by applying the channel estimation at receiver. Next, it is passed to transmitters using the feedback loops between the receivers and the transmitters.

To make the ad hoc network fully cooperative another assumption is made that each of the $N$ nodes not only feeds information about the channel properties but also provides the information about the interference to the desired transmitting node. Under these conditions, the desired transmitting node applies $N$ different signal transmission schemes to $N$ receiving nodes to maximize the mutual information (channel capacity) to each node. This strategy leads to optimization of the overall capacity of ad hoc network.

In order to obtain the capacity of the cooperative network, first we introduce the combined channel correlation in terms of channel $\mathbf{H}_{i j}$ as,

$$
\begin{aligned}
R_{\text {comb }} & =\mathbf{H}_{i j}{ }^{H} R_{N_{I}}^{-1} \mathbf{H}_{i j} \\
& =\left(R_{R m u}^{i j} G_{H_{i j}} R_{T m u}^{i j}\right)^{H} R_{N_{i}}^{-1}\left(R_{R m u}^{i j} G_{H_{i j}} R_{T m u}^{i j}\right)
\end{aligned}
$$

In order to optimize the channel capacity between node $i$ and the desired transmitting node $j$ a water-filling algorithm can be applied. Using this algorithm, the optimized channel capacity can be expressed as:

$$
C_{i}^{o p t}=\sum_{m=1}^{M_{r t}} \log _{2}\left(1+\frac{p_{m}^{i j}}{\sigma_{n}^{2}} \lambda_{m}^{i j}\right)
$$

in which $p_{m}{ }^{i j}$ is the optimal transmitted power from the desired transmitting node $j$ to node $i$, which is the subject to the condition

$$
P_{j}=\sum_{m=1}^{M_{r t}} p_{m}^{i j}
$$

$\lambda_{m}{ }^{i j}$ is the $m$-th eigenvalue of combined channel correlation $R_{\text {comb }}$ given by (25).

The optimal transmitted power at the $m$-th antenna is obtained using the following equation,

$$
\begin{aligned}
& p_{i j}=\left(\mu_{i j}-\frac{1}{\lambda_{m}^{i j}}\right)^{+} \\
& \text {subject to }: \sum_{m=1}^{M_{r t}} p_{m}^{i j}=P_{i j}
\end{aligned}
$$

in which $(\mathrm{x})^{+}=\max (0, \mathrm{x})$ and $\mu$ is chosen to obey the power constraint. The capacity of the cooperative ad hoc network with the optimized signal transmission scheme is given as,

$$
C^{o p t}=\sum_{i=1}^{N} C_{i}^{o p t}=\sum_{i=1}^{N} \sum_{m=1}^{M_{r t}} \log _{2}\left(1+\frac{p_{m}^{i j}}{\sigma_{n}^{2}} \lambda_{m}^{i j}\right)
$$

\section{Simulation Results}

In this section, computer simulations are performed to investigate the effects of spatial correlation and mutual coupling on capacity of ad hoc network. In the undertaken simulations, each node of ad hoc network is assumed to be equipped with a 4-element uniform array antenna. The elements are assumed to be wire dipoles having length of $0.5 \lambda$, where $\lambda$ is the carrier wavelength. The transmit/receive spatial correlation matrices for each pair of nodes are obtained using Equations (9) and (10) as presented in Subsection 2.2. The mutual coupling matrices are obtained from expressions (17) and (18) as given in Subsection 4.3.

Figure 2 presents the ad hoc network capacity in a 2-dimentional (2D) manner. The $\mathrm{X}$ and $\mathrm{Y}$ axis represent the capacity as an empirical distribution function (EDF), which is a cumulative probability $1 / M$ at each of the $M$ numbers in a sample,

$$
F_{M}(x)=\frac{X_{i} \leq x}{M}=\frac{1}{M} \sum_{i=1}^{M} I\left(X_{i} \leq x\right), i=1, \ldots, M
$$

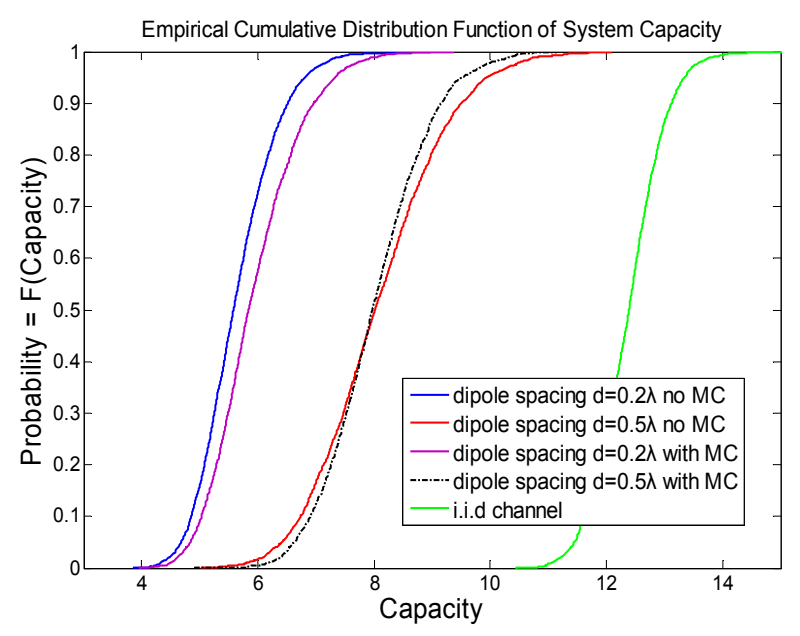

Figure 2. The empirical distribution function (EDF) of capacity of single user vs multiple user MIMO system. 
where $X_{i}$ is the $i$-th element in the sample and $I(A)$ is the indicator of event $A$. The $\mathrm{X}$-axis represents the capacity while $\mathrm{Y}$-axis indicates probability.

It can be seen in Figure 2 that when the spatial correlation exists, the capacity of ad hoc network is lower than when an ideal independent identical distribution (i.i.d) channel is assumed. This confirms the findings already obtained for single-user and multi-access MIMO systems reported in [8].

The presented results also show that when the dipole antenna spacing is small and equal to $0.2 \lambda$, the capacity with mutual coupling effect is larger compared to the one when mutual coupling is neglected. When the spacing is increased to $0.5 \lambda$ the capacity curves, for with and without mutual coupling effect, are getting very close to each other. The gap between these curves is getting smaller.

The results obtained for the 4-element linear wire dipole arrays indicate that at the element spacing of $0.2 \lambda$, mutual coupling decreases spatial correlation level and improves capacity. When the spacing becomes larger, the mutual coupling effect becomes less pronounced and there is not much difference in capacity results when the mutual coupling is neglected or taken into consideration.

Figure 3 presents the ad hoc network capacity in a 3-dimentional (3D) manner. The $\mathrm{X}$ and $\mathrm{Y}$ axes represent the capacity as an empirical distribution function (EDF), similarly as in Figure 2. In comparison with Figure 2, the $\mathrm{Z}$ axis is added to indicate the antenna spacing between the adjacent dipoles. There are two surfaces representing the ad hoc network capacity. One surface represents the case of antenna arrays without mutual coupling while the other one stands for the case when the mutual coupling effect is included in calculations. From the results presented in Figure 3, one can see that when the dipole spacing is within $0.2 \lambda$ to $0.4 \lambda$, at a fixed high probability, the capacity of ad hoc network with mutual coupling effect is higher than when mutual coupling is not taken into account. The trend becomes opposite when the spacing gets larger and is in the range of $0.4 \lambda$ to $0.6 \lambda$. A cross-point occurs at the spacing equal to $0.4 \lambda$. For the element spacing of $0.6 \lambda$ to $1 \lambda$, two surfaces overlap. The observed trends indicate that when the spacing is small $(0.2 \lambda$ to $0.4 \lambda)$, the mutual coupling decreases spatial correlation and improves capacity. When the spacing is in the range of $0.4 \lambda$ to $0.6 \lambda$, the mutual coupling increases the spatial correlation and decreases capacity. When the spacing exceeds $0.6 \lambda$, the mutual coupling weakly affects capacity and thus can be neglected because two sets of results without and with mutual coupling are almost identical.

Figure 4 shows the results similar to those of Figure 3 but for the case of non-optimized and optimized signal transmission schemes as given by expression (24) and (29), respectively.

The results shown in Figure 4 reveal that signal transmission optimization significantly improves ad hoc network capacity for both cases when mutual coupling is neglected or taken into account. The gap between the two capacity surfaces, with and without mutual coupling, is larger than for the non-optimized transmission scheme presented in Figure 3.

The differences between the non-optimized (expression (24))

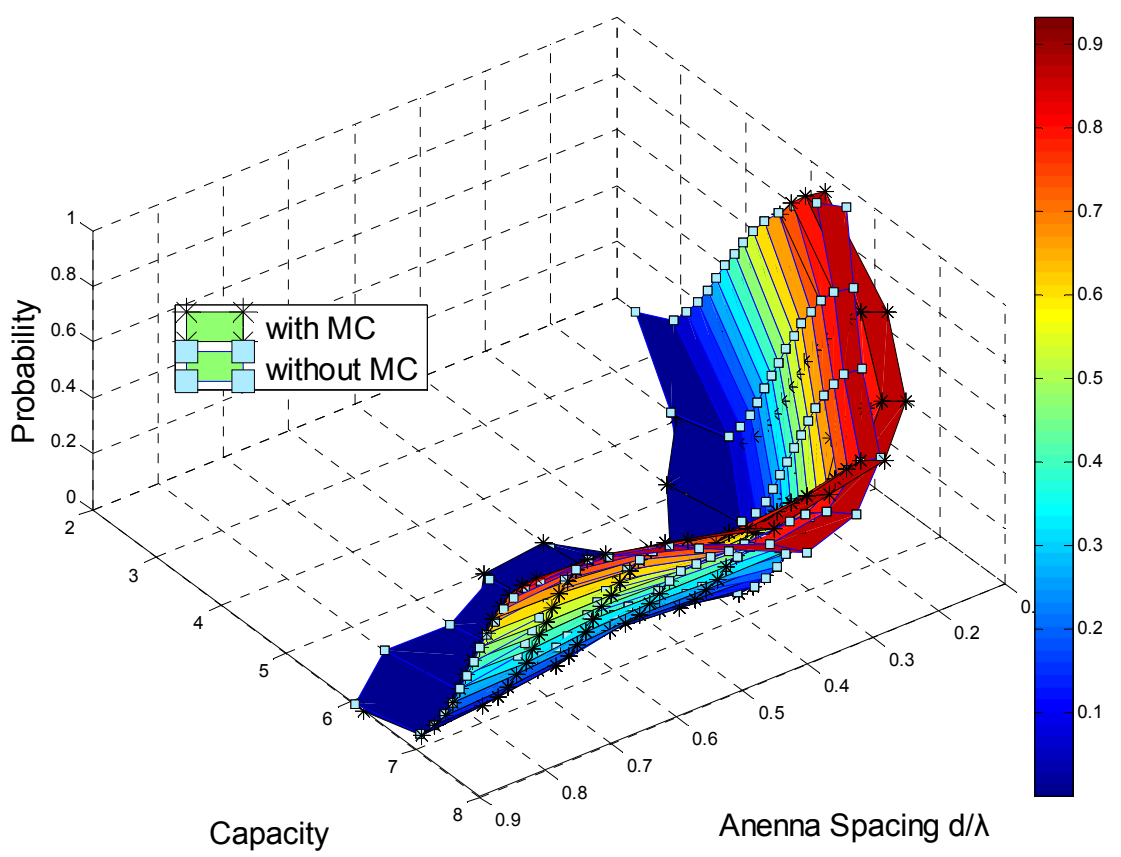

Figure 3. The empirical distribution function (EDF) of capacity vs Antenna spacing with and without mutual coupling (MC) 


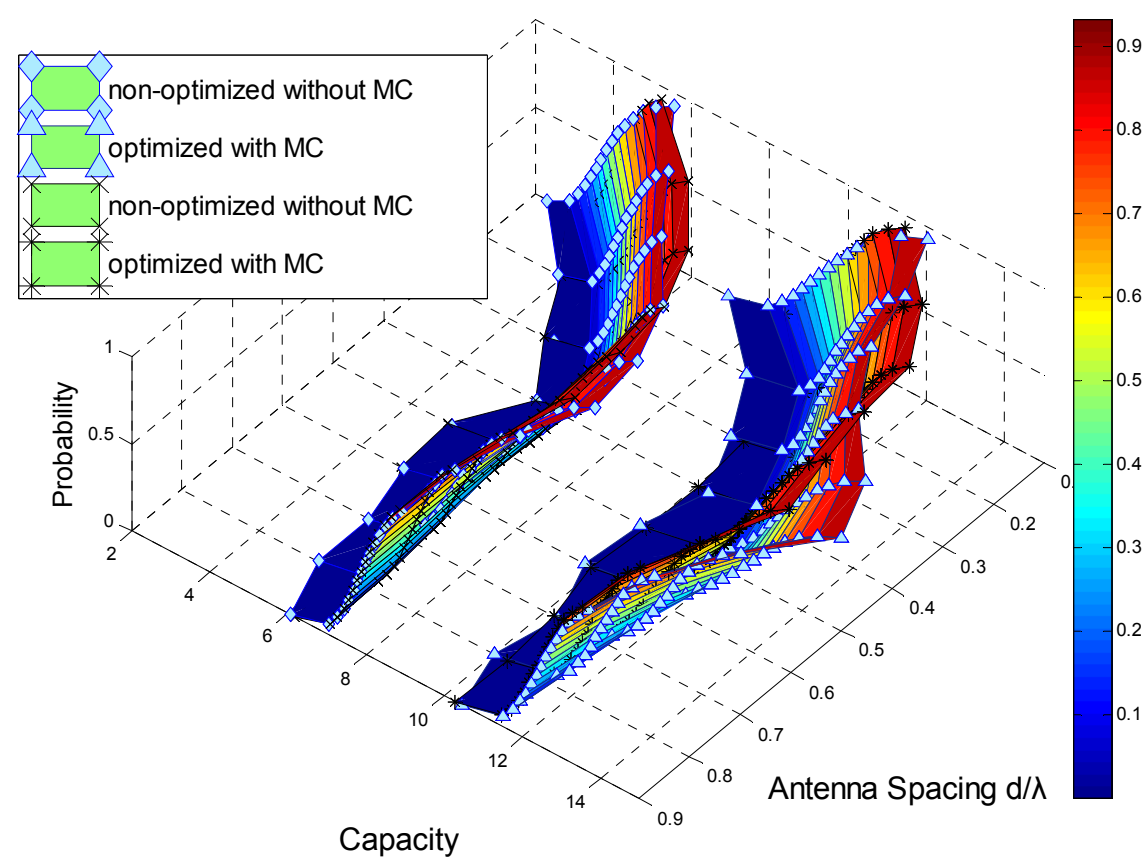

Figure 4. Non-optimized and optimized capacity vs Antenna spacing with and without mutual coupling (MC)

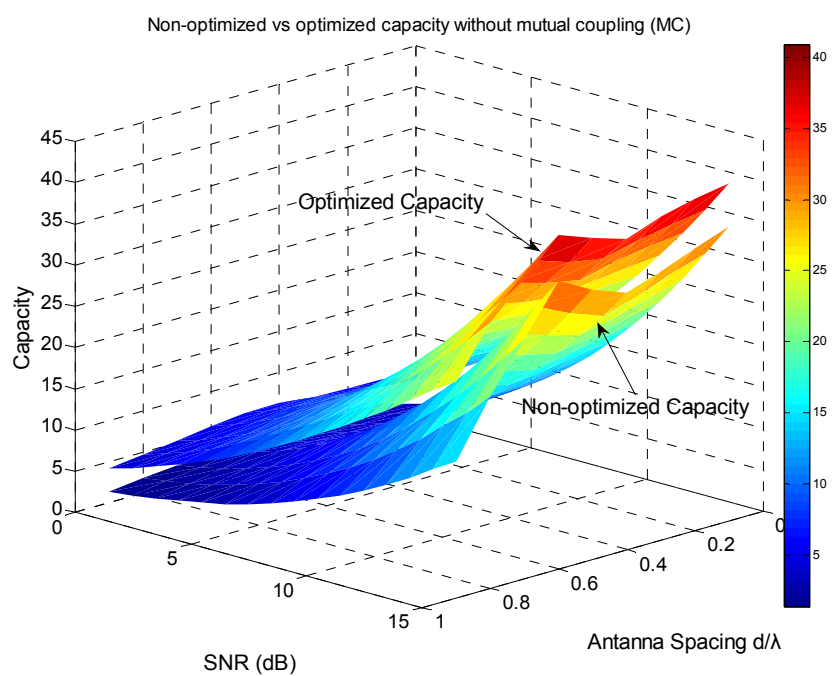

A. Non-optimized vs optimized capacity without MC

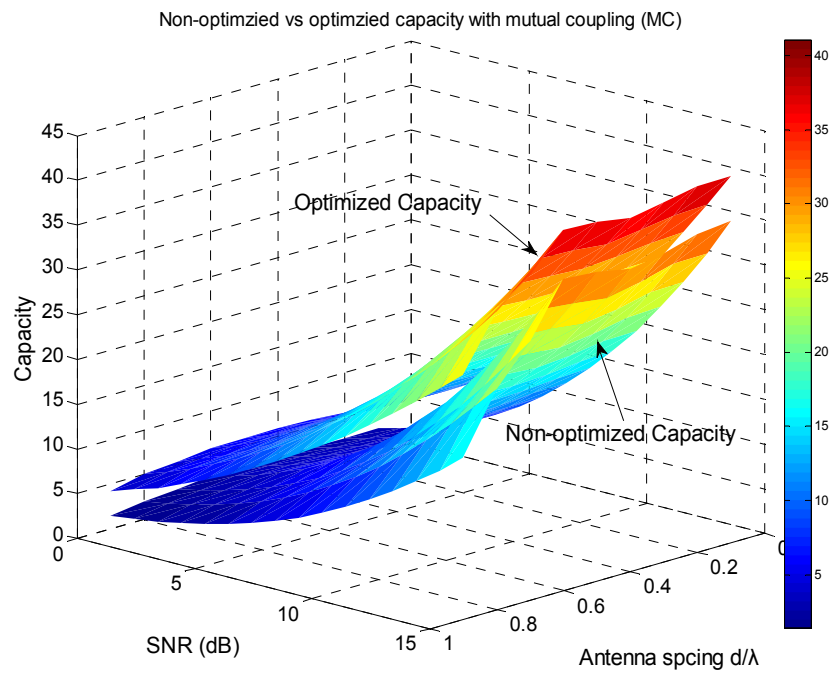

B. Non-optimized vs optimized capacity with MC

Figure 5. Non-optimized vs optimized ergodic capacity with and without mutual coupling.

and optimized (expression (29)) signal transmission cases can also be investigated in terms of ergodic capacity.

Figure 5 presents in the $3 \mathrm{D}$ manner the results for ergodic capacity versus antenna spacing (from $0.1 \lambda$ to $1 \lambda$ ) and SNR (from 0 to $15 \mathrm{~dB}$ ) for non-optimized as given by expression (29) and optimized as given by expression (24) signal transmission schemes.

Figure $5 \mathrm{~A}$ presents the results when the mutual coupling effect is neglected, while Figure 5B shows the results when the mutual coupling effect is taken into ac- count. One can see from the two Figures that irrespective of including or neglecting mutual coupling, the capacity for the optimized signal transmission scheme is higher than for the non-optimized case.

Figure 6 is another representation of results shown in Figure 5 when the signal transmission scheme is nonoptimized.

Figure 6A presents the capacity without mutual coupling while Figure $6 \mathrm{~B}$ presents the capacity when the mutual coupling effect is taken into account. One can see at spacing from $0.1 \lambda$ to $0.4 \lambda$, that the capacity under mutual 


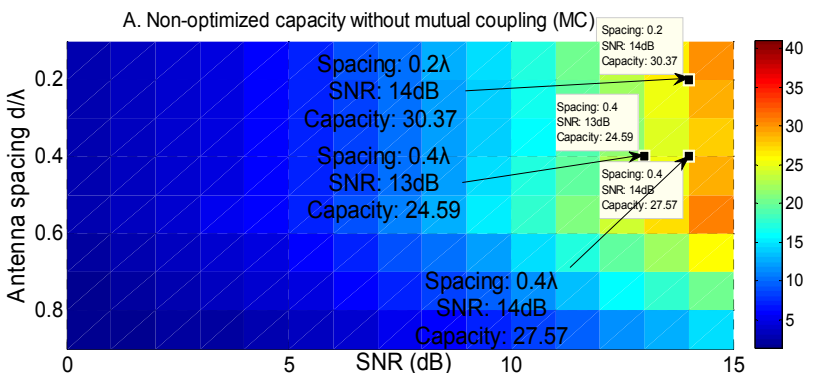

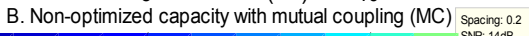

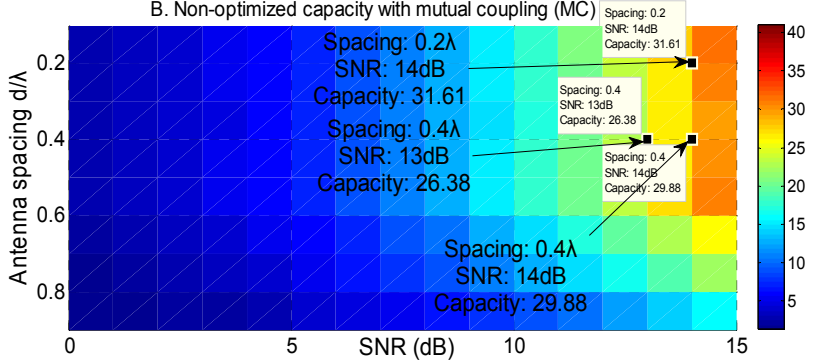

Figure 6. 2-D view of non-optimized ergodic capacity without (A) and with (B) mutual coupling 2-D view.
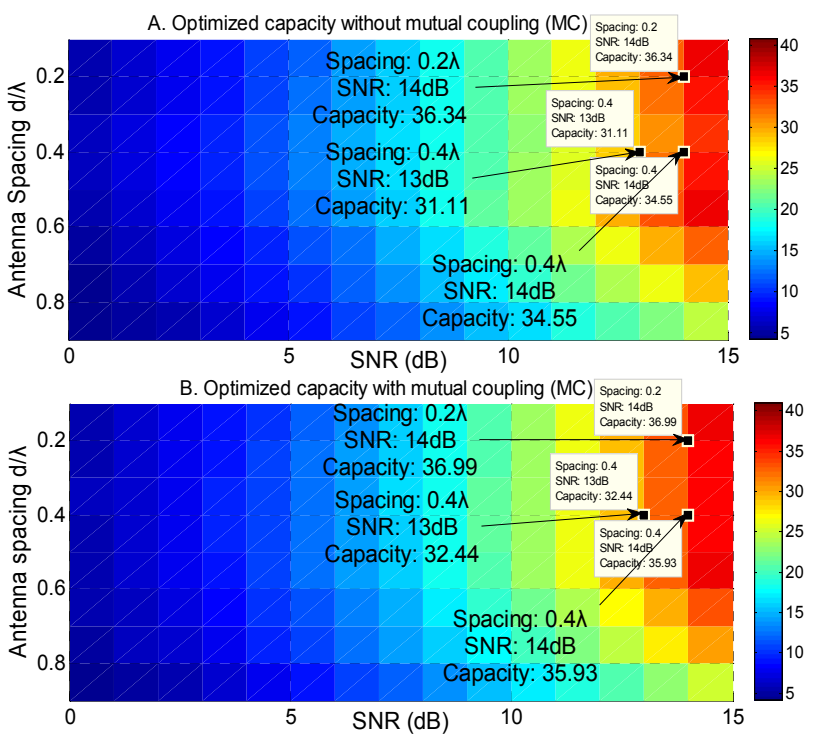

Figure 7. Optimized ergodic capacity without (A) and with (B) mutual coupling (MC) 2-D view.

coupling effect is higher than the one with no mutual coupling consideration. This property is more apparent at higher values of SNR.

Similarly, Figure 7 provides another representation of results shown in Figure 5 for the case when the signal transmission scheme is optimized. Figure 7A presents the capacity when mutual coupling is neglected while Figure 7B presents the capacity with the mutual coupling effect taken into account. One can see that at spacing between $0.1 \lambda$ to $0.4 \lambda$ the capacity under mutual coupling effect is higher than the one with no mutual coupling consideration. However, when the spacing is larger then the cross point of $0.4 \lambda$, the effect of mutual coupling is unnoticeable.

\section{Conclusions}

This paper has reported on investigations into the capacity of narrowband MIMO ad hoc network, in which nodes are equipped with multiple element antennas in the form of wire dipoles. The investigations have included the effect of mutual coupling in addition to spatial correlation that is present in the nodes array antennas. The spatial correlation has been taken into account using the Kronecker representation of the channel. Mutual coupling has been included using the closed-form expressions for impedance matrices of parallel side-to-side dipoles. Two cases of non-optimized and optimized signal transmission scheme have been considered. In the optimized signal transmission scheme the cooperative ad hoc network has been assumed, in which desired CSI and interference CSI are available at all the transmitting nodes. The computer simulations have been carried out for the case when the nodes are equipped with four- element uniform half-wave dipole arrays surrounded by circles of uniformly distributed scattering objects. The obtained simulation results for small size array antennas (of 4 elements) have shown that the spatial correlation decreases the capacity. At the antenna (dipole) spacing between $0.1 \lambda$ to $0.4 \lambda$, mutual coupling decreases the spatial correlation and helps to improve the capacity of MIMO ad hoc network. When the spacing is in the range of $0.4 \lambda$ to $0.6 \lambda$ or exceeds $0.6 \lambda$ the capacity is almost the same when the mutual coupling is taken into account or neglected. Assuming that the ad hoc network is cooperative, it has been shown that its capacity can be significantly improved by applying the optimized signal transmission scheme. The significant improvement has been demonstrated irrespectively whether the mutual coupling effects are neglected or taken into account.

\section{References}

[1] G. J. Foschini, "Layered space-time architecture for wireless communication in a fading environment when using multielement antennas," Bell Labs Technical Journal, Vol. 1, pp. 41-59, 1985.

[2] G. J. Foschini and M. J. Gans, "On limits of wireless communications in a fading environment when using multiple antennas," Wireless Personal Communications, Vol. 6, pp. 311-335, 1998.

[3] E. Telatar, "Capacity of multi-antenna Gaussian channels," European Transactions on Telecommunications, Vol. 10, No. 6, pp. 585-596, November 1999.

[4] B. Chen and M. J. Gans, "MIMO communications in ad hoc networks," IEEE Transactions on Signal Processing, Vol. 54, No. 7, Juy 2006.

[5] K. Fakih, J. Diouris, and G. Andrieux, "On the capacity optimization for MIMO ad hoc networks," IEEE VTC 
Spring, Singapore, pp. 1087-1091, May, 2008.

[6] D. Shiu, J. Foschini, M. J. Gans, and J. M. Kahn, "Fading correlation and its effect on the capacity of multielemnt antenna system," IEEE Transactions on Communication, Vol. 48, No. 3, March 2000.

[7] X. Liu and M. E. Bialkowski, "Effect of channel estimation on capacity of MIMO system employing circular or linear receiving array antennas," International Journal of Electronics, Communications and Computer Engineering, Vol. 1, No. 1, pp. 61-71, 2009.

[8] X. Liu, F. Wang, K. Bialkowski, and M. E. Bialkowski, "On the capacity of MIMO system operating in multiaccess scenario," to appear in Proceedings of APMC09 Singapore, pp. 7010, December, 2009.

[9] T. Svantesson and A. Ranheim, "Mutual coupling effects on the capacity of multi-element antenna systems," Proceedings of ICASSP01, pp. 2485-2488, April 2001.

[10] J. W. Wallace and M. A. Jensen, "The capacity of MIMO wireless systems with mutual coupling," IEEE VTC02, Vancouver, Canada, pp. 696-700, 2002.

[11] J. Wallace and M. Jensen, "Mutual coupling in MIMO wireless systems: A rigorous network theory analysis," IEEE Transactions on Wireless Communications, pp. 1317-1325, March 2004.
[12] P. N. Fletcher, M. Dean, and A. R. Nix, "Mutual coupling in multielement array antennas and its influence on MIMO channel capacity," IEEE Electron Letters, No. 39, pp. 342-344, 2003.

[13] E. G. Larsson and P. Stoica, "Space-time block coding for wireless communication," Cambridge University Press, 2003.

[14] C. N. Chuah, D. N. C. Tse, and J. M. Kahn, "Capacity scaling in MIMO wireless systems under correlated fading," IEEE Transactions on Information Theory, Vol. 48, pp. 637-650, March 2002.

[15] M. Medard, "The effect upon channel capacity in wireless communications of perfect and imperfect knowledge of the channel," IEEE Transactions on Information Theory, Vol. 46, No. 3, pp. 933-946, May 2000.

[16] S. Durrani and M. E. Bialkowski, "Effect of mutual coupling on the interference rejection capabilities of linear and circular arrays in CDMA systems," IEEE Transactions on Antennas and Propagation, Vol. 52, No. 4, pp. 1130-1134, April 2004.

[17] M. E. Bialkowski, P. Uthansakul, K. Bialkowski, and S. Durrani, "Investigating the performance of MIMO systems from an electromagnetic perspective," Microwave and Optical Technology Letters, Vol. 48, No. 7, pp. 1233-1238, July 2006. 